\title{
Descripción anatómica del abordaje anterior de cadera: seguridad y efecto para artroplastía total
}

\author{
Anatomical description of the anterior hip approach: safety and effect for total arthroplasty \\ Diego-Ball D, * Cabal AA ${ }^{\ddagger}$
}

Unidad Médica de Alta Especialidad, Hospital de Traumatología y Ortopedia Lomas Verdes del Instituto Mexicano del Seguro Social.

RESUMEN. Introducción: El abordaje anterior de cadera fue descrito en 1881, desde entonces se han realizado diversos estudios que han demostrado ventajas significativas frente a los abordajes posterior y lateral directo de cadera. Material y método: Se llevó a cabo un estudio descriptivo con casos continuos no probabilísticos en el Instituto de Ciencias Forenses de Octubre de 2015 a Julio de 2017. Se describió anatomía y distancias a los paquetes vasculonerviosos. Se realizó correlación de Pearson y Rho de Spearman. Resultados: Se efectuaron 22 disecciones, el nervio femorocutáneo fue identificado en nueve especímenes, la distancia promedio del nervio femorocutáneo lateral al intervalo de Smith-Petersen fue $11.4 \mathrm{~mm}$, se identificó la arteria circunfleja lateral ascendente debajo del recto femoral hacia la región central del abordaje, se colocaron los separadores alrededor de la articulación coxofemoral sin lesionar estructuras vitales, el separador más riesgoso se ubicó en la pared anterior del acetábulo, debajo del músculo iliopsoas con distancia promedio de $28.25 \mathrm{~mm}$ al paquete femoral. A mayor edad mayor distancia a los paquetes neurovasculares $\mathrm{p}<0.05$. Conclusiones: Alto nivel de seguridad del abordaje anterior para artroplastía de cadera, las distancias a estructuras vitales presentan un margen razonable, en artroplastía de cadera ofrece adecuada exposición de la articulación, visión directa del acetábulo y disección quirúrgica atraumática.

Palabras clave: Cadera, abordaje, anterior, seguridad, cadáver, prótesis.
ABSTRACT. Introduction: The anterior hip approach was described since 1881, since then several studies have been conducted that have shown significant advantages over the posterior and lateral direct approaches of the hip. Material and method: We conducted a descriptive study with continuous non-probabilistic cases at the Institute of Forensic Sciences from October 2015 to July 2017. Anatomy and distances were described to the neurovascular bundles. Correlation of Spearman's Pearson and Rho was performed. Results: 22 dissections were made, the Femorocutaneous Nerve was identified in 9 specimens, the average lateral Femorocutaneous Nerve distance at SmithPetersen interval was $11.4 \mathrm{~mm}$, We identified the Ascending Lateral Circumflex artery under the femoral rectum towards the central region of the approach, the separators could be placed around the coxofemoral joint without injuring vital structures, the riskier separator we place it in the anterior wall of the acetabulum, below the Psoasyland with an average distance $28.25 \mathrm{~mm}$ to the femoral package. The older you go, the longer the neurovascular bundles were located $\mathrm{p}<0.05$. Conclusions: High level of safety of the previous approach for hip replacement, distances to vital structures have a reasonable margin, hip replacement offers adequate joint exposure, direct acetabulum vision and atraumatic surgical dissection.

Keywords: Hip, surgical approach, anterior, safety, cadaver, prosthetics.

\section{Nivel de evidencia: IV}

* Médico adscrito del Centro Médico ABC Santa Fe, Ciudad de México, México.

${ }^{\ddagger}$ Cirujano Ortopedista y Traumatólogo.

Dirección para correspondencia:

Dr. Daniel Diego-Ball

E-mail: diego.ortopediacentro@gmail.com

https://dx.doi.org/10.35366/95327 doi: 10.35366/95327 


\section{Introducción}

El abordaje anterior directo de cadera (AAC) fue descrito por Carl Hueter en 1881, al cual se le atribuye la primera publicación, ${ }^{1,2}$ lo describía tomando como referencia la espina ilíaca anterosuperior y la punta del trocánter, a la mitad de esa distancia partía su abordaje y se dirigía distalmente de manera paralela al borde lateral del sartorio, desinsertando de manera parcial las fibras proximales del vasto lateral. ${ }^{2}$ Sin embargo, fue Smith-Petersen el que desarrolla esta técnica desde 1917 con su prolífica descripción del intervalo entre los músculos sartorio y tensor de la fascia lata extendiéndose a lo largo de la cresta ilíaca para una mejor exposición del acetábulo., ${ }^{2,3}$

La primera artroplastía de cadera mediante un AAC la ejecutó por primera vez Robert Judet en el año de 1947 y Charnley en los años $60{ }^{1,2}$ A lo largo de la historia diferentes procedimientos se han realizado mediante AAC, desde el tratamiento de tuberculosis de la cadera, ${ }^{2,4}$ displasias de la cadera por Salter, ${ }^{2,5}$ tratamiento de la necrosis avascular mediante la colocación de injerto óseo y tratamiento de facturas en la cabeza femoral. ${ }^{6,7}$

En la actualidad los abordajes más usados para la cadera son el abordaje lateral de Hardinge y el posterior, para los cuales se realiza disección de músculos rotadores con el riesgo de denervación parcial, en el caso de abordaje posterior y desinserción del glúteo medio con el riesgo de cicatrización incompleta resulta en muchos casos en debilidad de los abductores y notable cojera (marcha Trendelenburg) ${ }^{8}$ en el caso del abordaje de Hardinge.

El AAC tiene ventajas estadísticamente significativas en lo que respecta a una mejor recuperación funcional temprana, ${ }^{9}$ los estudios indican que según la escala de Harris el AAC es la técnica más efectiva en las primeras cuatro a seis semanas y en el último seguimiento de la escala cuando es comparado con el abordaje lateral y posterior con un puntaje de 92.9 en la escala de Harris. ${ }^{10,11}$

En cuanto a la incidencia de luxación protésica sobre el abordaje posterior y lateral directo; Serialli y colaboradores reportaron que la mayor incidencia de luxación protésica se presenta con abordaje posterior $4 \%$, el abordaje anterolateral presenta $2 \%$ y el AAC de 0.6 a $1 \%{ }^{12,13,14,15}$

En cuanto al tiempo de hospitalización, éste se reduce en 1.19 días en comparación con los pacientes operados mediante abordaje anterolateral y posterior, produciendo menor costo operativo; sin embargo, el índice de transfusiones fue similar entre los tres grupos así como los análisis radiográficos en cuanto a inclinación y anteversión del componente femoral. ${ }^{13,15,16}$

En cuanto a la curva de aprendizaje, investigadores de la Universidad de Melbourne determinaron que un cirujano debe realizar al menos 50 procedimientos para que el rango de cirugías de revisión no sea diferente al del cirujano que ha realizado 100 o más procedimientos, es así que el rango de cirugías de revisión por diversos motivos en cirujanos noveles (principiantes, de poca experiencia) de $6 \%$ y el ci- rujano experimentado con más de 100 procedimientos es de $3 \% .^{17,18}$

Una de las complicaciones más frecuentes es la lesión del nervio femorocutáneo lateral (de 0.3 a $2.6 \%$ ), este riesgo se puede disminuir significativamente centrando la incisión sobre el vientre del músculo tensor de la fascia lata. ${ }^{19} \mathrm{La}$ lesión del nervio femorocutáneo lateral trae como complicación principal la meralgia parestética, en la cual el paciente presenta adormecimiento, quemazón o dolor sobre la superficie anterolateral del muslo. ${ }^{20,21,22}$

El AAC para prótesis total es una técnica que ha tomado mucha aceptación en los últimos años, esto se debe principalmente a que ha presentado varias ventajas con respecto a abordajes convencionales, sobre todo en lo que respecta a una recuperación funcional temprana, disminución de complicaciones como la marcha de Trendelenburg y a menores índices de luxación protésica y de sangrado transquirúrgico. ${ }^{10,11,12,13,14,15}$

Actualmente en México no existen estudios académicos que demuestren la seguridad de este procedimiento, por tal motivo hemos decidido realizar un estudio anatómico de las estructuras implicadas en este abordaje así como determinar los riesgos implicados en el mismo y el efecto en la técnica para artroplastía total de cadera.

\section{Material y método}

El presente estudio se llevó a cabo en el Departamento de Investigación en Salud de La Unidad Médica de Alta Especialidad Hospital de Traumatología y Ortopedia Lomas Verdes del Instituto Mexicano del Seguro Social con el permiso de las autoridades del Instituto de Ciencias Forenses (INCIFO) en Ciudad de México.

Se realizó un estudio descriptivo con casos continuos no probabilísticos desde Octubre de 2015 a Julio de 2017, se describió anatomía y hallazgos característicos, capacidad de realizar capsulotomía, luxación de articulación coxofemoral y distancias a los paquetes vasculonerviosos. Se disecaron 11 cadáveres proporcionados por el Instituto de Ciencias Forenses, en los cuales se realizaron 22 disecciones con los siguientes criterios de inclusión: cadáveres del sexo mascu-

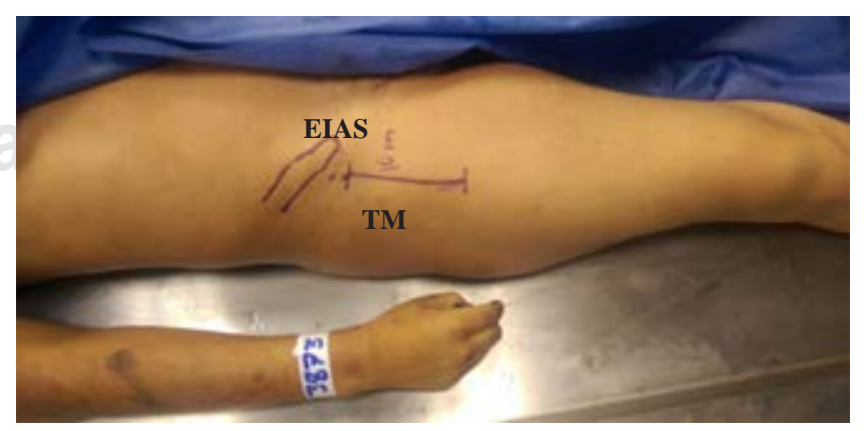

Figura 1: Marcaje quirúrgico, tomando como referencia la espina ilíaca anterosuperior (EIAS), trocánter mayor (TM) y vientre muscular del tensor de la fascia lata. 


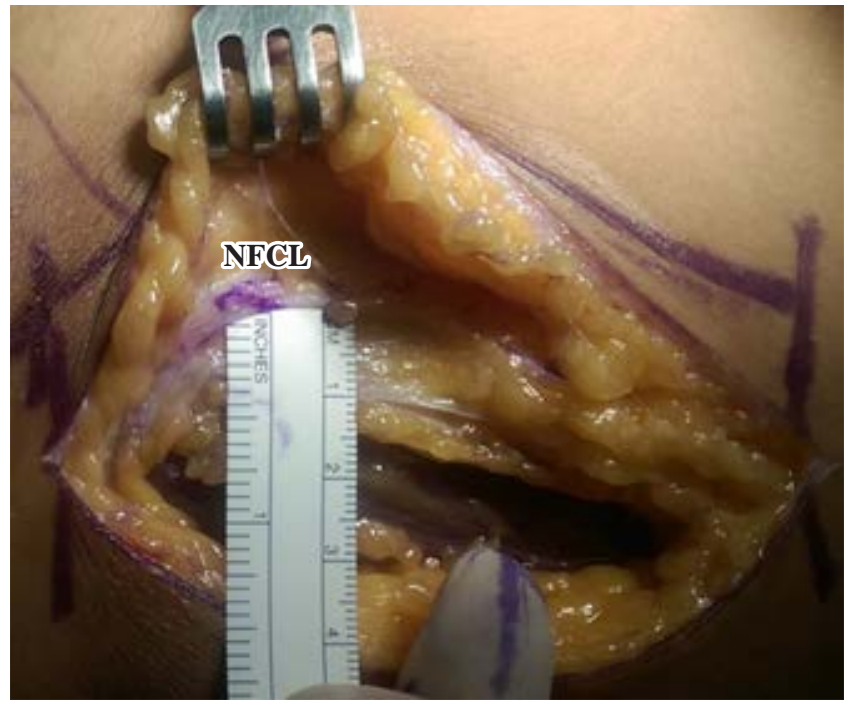

Figura 2: Nervio femorocutáneo lateral.

lino o femenino, mayores de 18 años de edad al momento del fallecimiento, con tiempo de fallecimiento menor de siete días.

Criterios de exclusión: cadáveres que presenten mutilaciones en extremidades inferiores, registro de procedimientos previos a nivel de la cadera.

Abordaje: puntos de referencia: espina ilíaca anterosuperior. Músculo tensor de la fascia lata cuando es palpable y borde lateral de la patela o cabeza del peroné. Desde la espina ilíaca anterosuperior $3 \mathrm{~cm}$ a distal y $3 \mathrm{~cm}$ a lateral es el punto más proximal de la incisión cutánea, desde allí trazamos una línea oblicua con orientación hacia el borde lateral de la patela o cabeza del peroné de $12 \mathrm{~cm}$ de extensión aproximadamente, es el punto más distal de la incisión cutánea (Figura 1).

Se incide la piel, se corta tejido celular subcutáneo hasta la fascia del tensor de la fascia lata, en este punto es muy importante diferenciar entre la fascia del músculo sartorio y tensor de la fascia lata, entre estos dos músculos está el intervalo de Smith-Petersen. Sugerimos que no se acceda al siguiente plano a través de este intervalo, ya que el nervio femorocutáneo lateral corre un alto riesgo de ser lesionado, recomendamos que se incida la fascia sobre el músculo tensor de la fascia lata en la mitad de su vientre muscular, desde este punto el nervio femorocutáneo lateral suele correr de forma longitudinal a unos $15 \mathrm{~mm}$, en 19 de las 22 disecciones realizadas se pudo identificar plenamente el nervio femorocutáneo lateral (Figura 2). Se continúa separando el vientre muscular del tensor de la fascia lata de su fascia anterior, se separa hacia medial tomando la fascia del tensor de la fascia lata junto con el músculo sartorio como protección del paquete vasculonervioso traccionando con un retractor de Meyerding hacia medial, luego se accede al espacio que se formó entre el vientre muscular del tensor de la fascia lata hacia lateral y el recto femoral a medial.
Se procede entonces a localizar la arteria circunfleja lateral ascendente, la cual se ubica en el punto medio de la longitud del abordaje y está cubierta por tejido adiposo ( $F i$ gura 3), procediendo a ligarla, cortarla o cauterizarla conjuntamente con sus venas satélites, se encuentra distal a esta arteria la porción profunda de la banda iliotibial, la cual se procede a disecar. Se accede a la cápsula articular de la cadera y se colocan tres separadores para exponer y delimitar adecuadamente el área de trabajo. Primero se coloca separador Hohmann a nivel medial del cuello, otro Hohmann a nivel lateral del cuello tomando como referencia la silla de montar que se forma en este punto sobre el trocánter mayor y la región cervical. Se coloca otro separador Hohmann a nivel de la cabeza femoral, hay que tomar en cuenta que los tres separadores son extracapsulares hasta este punto.

Se procede a realizar capsulotomía en $\mathrm{T}$ invertida o rectangular y se reseca la cápsula anterior, se recolocan los separadores en la misma ubicación anatómica, pero intracapsulares esta vez (Figura 4). Se realiza corte transcervical con sierra oscilante, el primer corte será subcapital transverso al eje del cuello y el segundo tomando como referencia el trocánter mayor de 50 grados de angulación, formando una pastilla ósea, la cual se extrae; se procede entonces a luxar el fémur con maniobra de aducción y rotación externa y se reorienta el corte transcervical quedando a 1-1.5 cm del trocánter menor y con inclinación de 50 grados.

Se procede a colocar un bulto debajo de la rodilla para hacer que el fémur se desplace a posterior y así mejorar la exposición del acetábulo, se recolocan separadores Hohmann para exponer el acetábulo, se coloca el primer retractor a nivel de la superficie posterior del acetábulo, otro separador en la pared anterior del acetábulo teniendo precaución de no colocarlo por encima del músculo iliopsoas ni en su espesor debido a que existe un riesgo muy alto de lesionar el nervio femoral y la arteria femoral, el punto más seguro es colocar este retractor debajo de las fibras del músculo

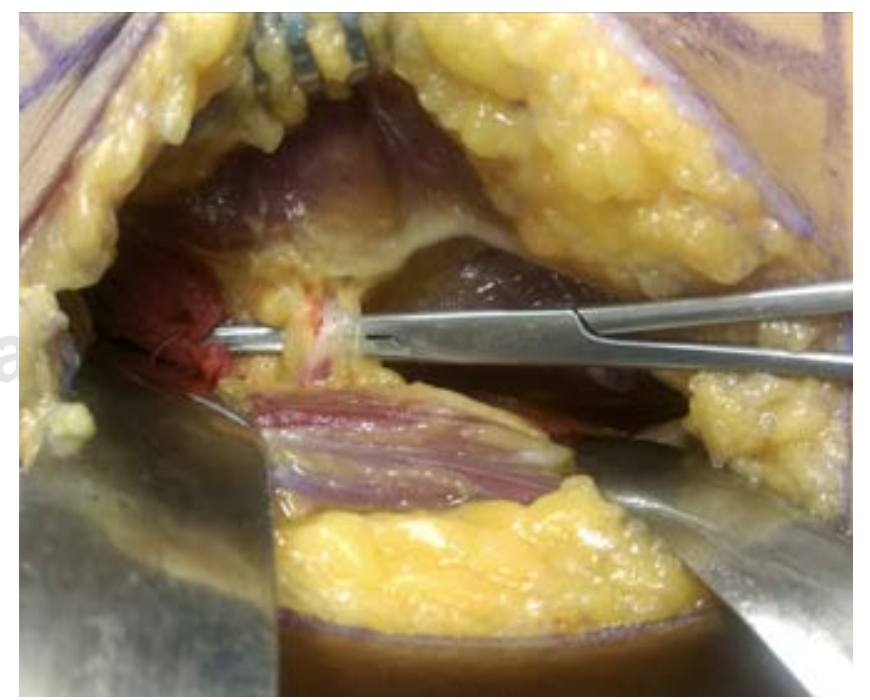

Figura 3: Arteria circunfleja lateral ascendente. 


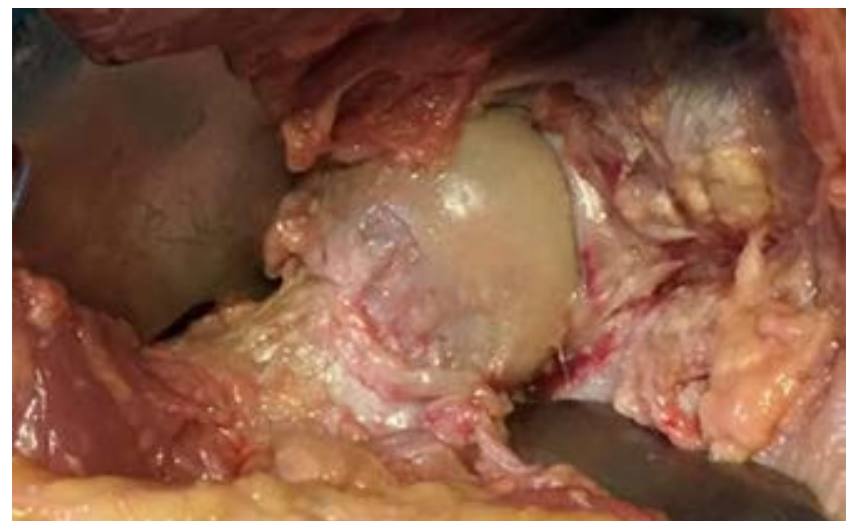

Figura 4: Exposición de la articulación coxofemoral luego de la capsulotomía.

iliopsoas, ya que al hacerlo de esta forma rechazamos el vientre muscular del iliopsoas y el paquete vasculonervioso a anterior y medial, cuando se realiza de esta manera el separador está a una distancia promedio de $3 \mathrm{~cm}$ del paquete vasculonervioso y finalmente se coloca otro separador tipo Müller o Hohmann en el piso del acetábulo, se procede entonces a extraer la cabeza femoral con ayuda de un tornillo de Schanz o un extractor tipo sacacorchos.

Se expone el acetábulo y se empieza su preparación con fresas acetabulares, tomando como referencia la espina ilíaca anterosuperior, el ligamento transverso y la horizontal de la mesa quirúrgica, se da la posición deseada y se coloca el componente acetabular definitivo, en este punto se puede tomar una radiografía AP de pelvis para constatar la orientación adecuada del componente acetabular y reorientar en caso de ser necesario.

Se procede a trabajar el fémur, para lo cual se da posición a la extremidad que se está operando, se da hiperextensión de las caderas así como aducción y rotación externa del miembro pélvico, al cual se le está realizando el procedimiento, se procede a colocar separadores Hohmann, el primer separador se coloca en el trocánter menor, puede ser un separador tipo Müller, se coloca un separador distal al trocánter menor para traccionar hacia lateral el fémur, se coloca un separador tipo Müller de dos picos por debajo del trocánter mayor para traccionar el fémur hacia anterior, se procede a trabajar el fémur, inicialmente con rima iniciadora de preferencia cola de ratón o localizador de canal, la forma de orientar es con el trocánter menor, se rima diafisario y metafisario y se procede a colocar el componente femoral definitivo de la medida que amerite, se comprueba estabilidad pasiva y se procede al cierre por planos de tejidos, se sutura la fascia del tensor de la fascia lata y piel.

Se recolectó toda la información de las disecciones y se condensó en la Tabla 1 tomando como puntos relevantes sexo, edad, fecha de disección, identificación del nervio femorocutáneo (NFC), distancia del NFC al intervalo de Smith-Petersen, identificación de la arteria circunfleja, capacidad de capsulotomía, capacidad de osteotomía cervical, capacidad de luxación femoral y la distancia del separador acetabular en la pared anterior del acetábulo al paquete neurovascular femoral.

Los datos se clasificaron en valores cuantitativos, categóricos y dicotómicos, se hizo estadística descriptiva de frecuencias y distribución de la muestra. Se realizó correlación de Pearson para variables cuantitativas y Rho de Spearman para variables categóricas o dicotómicas. Se tomó como significativo todo valor de $\mathrm{p}$ menor de 0.05 . Se encontró correlación entre las variables: edad y distancia del paquete neurovascular femoral ( $\mathrm{p}<0.05, \mathrm{r}=0.650$ ) a mayor edad del espécimen mayor distancia al paquete neurovascular femoral y mayor distancia desde el intervalo de Smith-Petersen al NFC.

\section{Resultados}

Se realizaron 22 disecciones, dentro de las cuales fueron 10 especímenes del sexo masculino y un espécimen femenino. El primer espécimen fue reservado exclusivamente para la disección e identificación anatómica de todas las estructuras implicadas en el abordaje (vasculares, nerviosas, musculares) de todo el muslo inclusive parte de la pelvis; el nervio femorocutáneo fue identificado en nueve de los 10 especímenes, siendo más frecuente del lado izquierdo $90 \%$, que el derecho $70 \%$, la distancia promedio del nervio femorocutáneo lateral al intervalo de SmithPetersen fue de $12.7 \mathrm{~mm}$ en las caderas izquierdas y de $10.7 \mathrm{~mm}$ en las caderas derechas, en todas las disecciones se pudo identificar la arteria circunfleja lateral ascendente $100 \%$, ésta se encontró invariablemente debajo del recto femoral hacia la región central del abordaje, en todas las disecciones se pudo exponer de manera adecuada la cápsula articular coxofemoral, luego se constató que se puede realizar capsulotomía en $\mathrm{T}$ invertida o rectangular sin dificultades, en todas las disecciones fue posible colocar los separadores alrededor de la articulación coxofemoral sin llegar a lesionar estructuras vitales, se determinó que el separador más riesgoso de colocar es el que está ubicado en la pared anterior del acetábulo; de este apartado se encontró que pueden existir dos posibilidades de colocación de este separador: debajo de las fibras musculotendinosas del músculo iliopsoas (85\%) con una distancia promedio de $29.5 \mathrm{~mm}$ al paquete femoral derecho y $27 \mathrm{~mm}$ al paquete femoral izquierdo, entre las fibras musculotendinosas del músculo iliopsoas (15\%) con una distancia promedio de 14 mm derecho y 10 mm izquierdo, también se determinó que el sitio más seguro para su colocación es por debajo de las fibras musculotendinosas del músculo iliopsoas con una distancia promedio del paquete neurovascular femoral de $28.25 \mathrm{~mm}$.

La exposición intracapsular se consideró adecuada, se realizó osteotomía doble, 17 de las 20 disecciones (85\% de las veces), en el primer espécimen no se efectuó osteotomía cervical porque se utilizó para identificación de las estructuras anatómicas de la cadera y muslo y en la última disección por dificultades técnicas del instrumental (sierra oscilante). 


\begin{tabular}{|c|c|c|c|c|c|}
\hline & $\mathrm{N}$ & Mínimo & Máximo & Media & Desv. típ. \\
\hline Edad & 10 & 25 & 64 & 51.20 & 12.656 \\
\hline Distancia del nervio a intervalo izquierdo & 10 & 0 & 18 & 12.70 & 5.100 \\
\hline Distancia del nervio a intervalo izquierdo & 10 & 0 & 18 & 10.10 & 7.295 \\
\hline Distancia del separador acetabular al paquete derecho & 10 & 13 & 35 & 26.40 & 8.435 \\
\hline Distancia del separador acetabular al paquete izquierdo & 10 & 10 & 35 & 25.30 & 7.288 \\
\hline N válido (según lista) & 10 & & & & \\
\hline
\end{tabular}

Hubo dificultad en una cadera, principalmente debido a un abordaje más distal del descrito en la técnica con dificultad de exposición del acetábulo, asimismo en este caso no fue posible luxar el fémur para trabajarlo de manera adecuada, se determinó que en situaciones en las que el fémur no se pueda luxar adecuadamente hacia anterior con las posiciones descritas en la técnica quirúrgica, es posible realizar liberación de la cápsula posterior e incluso liberar parte de la inserción de los rotadores, esto permite movilizar mejor el fémur y así luxarlo hacia anterior y trabajar el componente femoral de forma más cómoda. En todos los casos se pudo realizar un adecuado cierre de planos de tejidos blandos. Se encontró correlación entre las variables: edad y distancia del paquete neurovascular femoral ( $\mathrm{p}<0.05$, $\mathrm{r}$ $=0.650$ ) a mayor edad del espécimen mayor distancia al paquete neurovascular femoral y mayor distancia desde el intervalo de Smith-Petersen al NFC.

\section{Discusión}

El abordaje anterior de cadera es una técnica que ha ganado más popularidad dentro de los círculos de cirujanos ortopedistas, principalmente en EUA y Europa; dentro de las ventajas que ofrece se observa mejor estabilidad de tejidos, menor daño muscular y en general mejor desempeño en comparación con las otras técnicas para artroplastía total de cadera. ${ }^{12,13}$ En nuestra serie el abordaje anterior de cadera permite realizar todos los pasos necesarios para llevar a cabo una artroplastía total de cadera en un paciente, esto debido a que la exposición de la articulación coxofemoral no presentó lesiones en estructuras adyacentes. Se identificaron las estructuras anatómicas principales como el nervio femorocutáneo lateral y el paquete vasculonervioso femoral, evitando su lesión.

Las características del abordaje descrito nos permite inferir que es seguro realizar el abordaje anterior de cadera, a tal punto que iguala los parámetros de seguridad de los abordajes convencionales de cadera lateral y posterior, las distancias de los separadores a las estructuras vitales como los paquetes neurovasculares presentan un margen seguro, a la vez que no hubo lesión incidental de ninguna estructura vital; esto representa un efecto importante en el desarrollo de la intervención quirúrgica que se desea aplicar, la cual es la artroplastía total de cadera debido a que en los especímenes presenta una adecuada exposición del acetábulo, visualización y orientación de la anteversión e inclinación de la copa acetabular; no se consideró el uso de material quirúrgico especial para la exposición de la cadera.

Por otro lado, la exposición femoral es mucho más difícil, motivo por el cual se han desarrollado diferentes técnicas utilizando desde separadores especiales, aparatos de tracción del fémur y mesas de tracción. ${ }^{1,23}$ Se deben adoptar diversas medidas como la liberación de la cápsula posterior o la liberación selectiva de los rotadores a nivel del trocánter mayor (tendón conjunto) para poder luxar adecuadamente el fémur hacia adelante y de esta forma tener adecuados puntos de referencia para la orientación adecuada del componente femoral como es el trocánter menor, en este punto se puede anticipar que el mayor grado de dificultad para trabajar el fémur puede tributar en complicaciones transquirúrgicas, principalmente fracturas periprotésicas femorales. Es necesario continuar con el estudio anatómico y de disección, ya que se encontraron correlaciones positivas entre la edad y la distancia del separador de la pared anterior, lo cual sugiere una variación en aumento de la distancia que se modifica con la edad. Otro hallazgo en la correlación fue con la variable de osteotomía cervical con luxación femoral que indica que realizando la osteotomía se puede llevar a cabo la luxación de la cadera para proceder a colocar el implante.

Aunque no fue necesaria la utilización de instrumental especial para la exposición quirúrgica, recomendamos el uso de instrumental adecuado (separadores angulados tipo cobra, localizador de canal medular, etc.) para este procedimiento en pacientes vivos, esto con la finalidad de minimizar el tiempo quirúrgico, facilitar la exposición del campo quirúrgico y mejorar tanto la orientación como el posicionamiento de los componentes protésicos.

Asimismo, la experiencia de poder realizar este abordaje en un espécimen cadavérico nos ha dado una perspectiva fidedigna de la orientación tridimensional de la cadera, de la proximidad de estructuras vitales y de los márgenes de seguridad que debemos respetar al realizar este procedimiento.

\section{Conclusiones}

Este abordaje permitió una adecuada exposición de la articulación de la cadera, visión directa del acetábulo, de su disposición y orientación; con mínimo daño a estructuras musculares y tendinosas sin olvidar la posición supina del paciente en la mesa quirúrgica. 
Bibliografía

1. Matta JM. The anterior approach for total hip replacement, background and operative technique, hip and pelvis institute at Saint John's Health Center. Santa Mónica, California 2008.

2. Rachbauer F, Kain M. The history of the anterior approach to the hip. Orthop Clin North Am. 2009; 40(3): 311-20.

3. Conolly KP, Kamath AF. Direct anterior total hip arthroplasty: Literature review of variations in surgical technique. World J Orthop. 2016; 7(1): 38-43.

4. Kirkaldy-Willis WH. Isquio-femoral arthrodesis of the hip in tuberculosis. an anterior approach. J Bone Joint Surge. 1950; 32B: 187-92.

5. Salter RB. Innominate osteotomy in the treatment of congenital dislocation and subluxation of the hip. J Bone Joint Surg [Br]. 1961; 43-B: 518-39.

6. Iwata H, Torii S, Hasegawa Y, Itoh H, Mizuno M, Genda E, Kataoka Y. Indications and results of vascularized pedicle iliac bone graft in avascular necrosis of the femoral head. Clin Orthop Relat Res. 1993: 295: 281-8.

7. Cubbins WR, Callahan JJ, Scuderi CS. Fractures of the neck of the femur. Surg Gy Obstet. 1939; 68: 87.

8. Ola Hallert, Yan Li. The direct anterior approach: initial experience of a minimally invasive technique for total hip arthroplasty. J Orthop Surg Res. 2012; 7: 17.

9. Restrepo C, Parvizi J. Prospective randomized of two surgical approaches for total hip arthroplasty. J Arthroplasty. 2010; 25(5): 671-9.e1.

10. Putananon C, Tachinda H. Comparison of direct anterior, lateral, posterior and posterior-2 approaches in total hip arthroplasty: network meta-analysis. Eur J Orthop Surg Traumatol. 2018; 28(2): 255-67.

11. Yao R, Howard JL. Conjoint tendon release in direct anterior total hip arthroplasty: no impact on patient outcomes. Orthopaedics. 2017; 40(6): e971.
12. Horne PH, Olson SA. Direct anterior approach for total hip arthroplasty using the fracture table. Curr Rev Musculoskelet Med. 2011; 4(3): 139-45.

13. Mirza AJ, Lombardi AV Jr., Morris MJ, Berend KR. A mini-anterior approach to the hip for total joint replacement: optimising results: improving hip joint replacement outcomes. Bone Joint J. 2014; 96 B(11 Supple A): 32-5.

14. Sariali E, Leonard P. Dislocation after total hip arthroplasty using hueter anterior approach. J Arthroplasty. 2008; 23(2): 266-72.

15. Yue C, Kang P. Comparison of direct anterior and lateral approaches in total hip arthroplasty. Medicine. 2015; 94(50): e2126.

16. Chow J, Pearce S, Cho K, Walter W. Direct anterior approach using navigation improves accuracy of cup position compared to conventional posterior approach. Cureus. 2017; 9(7): e1482.

17. De Steiger RN, Lorimer M. What is the learning curve for anterior approach for total hip arthroplasty? Clin Orthop Relat Res. 2015; 473(12): 3860-6.

18. De Geest T, Fennema P. Adverse effects associated with the direct anterior approach for total hip arthroplasty: bayesian meta-analysis. Arch Orthop Trauma Surg. 2015; 135(8): 1183-92.

19. York PJ, Smarck CT. Total hip arthroplasty via anterior approach: tips tricks for primary and revision surgery. Int Orthop. 2016; 40(10): 2041-8.

20. Ropars M, Morandi X. Anatomical study of the lateral femoral cutaneus nerve with special reference to minimally invasive anterior approach for total hip replacement. Surg Radiol Anat J. 2009; 31(3): 199-204.

21. Aszmann OC, Dellon ES, Dellon AL. Anatomical course of the lateral femoral cutaneous nerve and its susceptibility to compression and injury. Plast Reconstr Surg. 1997; 100(3): 600-4.

22. Bjurlin MA, Davis KE, Allin EF, Ibrahim DT. Anatomic variations in the lateral femoral cutaneous nerve with respect to pediatric hip surgery. Am J Orthop (Belle Mead NJ). 2007; 36: 143-6.

23. Kain MS, Leunig M. Anterior approach for total hip arthroplasty: technique without fracture table, mini-invasive surgery of the hip. Bone Joint J. 2014; 96-B(11 Supple A): 32-5. 